\title{
Endoscopic Endonasal Repair of Internal Carotid Artery Injury during Endoscopic Endonasal Surgery
}

\author{
Irit Duek ${ }^{1,2}$ Gill E. Sviri ${ }^{3}$ Moran Amit ${ }^{1,2}$ Ziv Gil ${ }^{1,2}$ \\ ${ }^{1}$ Department of Otolaryngology Head and Neck Surgery, The Head \\ and Neck Center, Rambam Health Care Campus, Haifa, Israel \\ 2 The Laboratory for Applied Cancer Research, Clinical Research \\ Institute, Rambam Health Care Campus, Technion - Israel Institute \\ of Technology, Haifa, Israel \\ ${ }^{3}$ Department of Neurosurgery, Rambam Health Care Campus, Haifa, Israel
}

Address for correspondence Ziv Gil, MD, PhD, Department of Otolaryngology Head and Neck Surgery, Rambam Medical Center, Technion - Israel Institute of Technology, 8 Ha'Aliya Street, POB 9602, Haifa 31096, Israel (e-mail: ziv@baseofskull.org).

J Neurol Surg Rep 2017;78:e125-e128.

\begin{abstract}
Keywords

- internal carotid artery injury

- endoscopic endonasal skull base surgery

- intraoperative injury

Background Injury to the cavernous portion of the internal carotid artery (ICA) during endoscopic skull base surgery is a well-recognized rare complication that can be associated with high rates of morbidity and mortality. Many techniques have been suggested to manage ICA injury with varying degrees of success.

Objectives We provide a detailed technical description of an operative technique for endoscopic management of carotid artery injury.

Methods A case of ICA injury during endoscopic skull base surgery is presented. The immediate treatment measurements include: (1) early recognition of ICA injury, (2) briefing of the team and preparations, (3) packing, (4) harvesting of temporalis muscle patch, (5) placement of the muscle patch over the defect, and (6) gentle compression for 10 minutes. Results The technique facilitates quick repair and restores normal blood flow through the damaged artery. Exsanguination or the symptoms of stroke that may occur from prolonged occlusion of the ICA are therefore prevented.

Conclusion The proposed protocol is useful for the management of a potentially lifethreatening ICA injury.
\end{abstract}

\section{Introduction}

Intraoperative injury to the internal carotid artery (ICA) during endoscopic endonasal surgery (EES) of the skull base is a rare $(0.2-1 \%)$ complication that can potentially be associated with high rates of morbidity and mortality. ${ }^{1}$ The most common site of injury within the ICA is the cavernous segment, with leftsided injuries occurring more frequently than right-sided injuries. Traumatic injury to the ICA during EES may cause massive hemorrhaging, leading to hypotension and shock. Massive hemorrhage can be difficult to control due to limited access to the sphenoid sinus and rapid obscuring of the visual field. Bleeding from this vital artery can become lethal within minutes. Even when the bleeding is controlled, perma- nent neurological deficits (such as altered mental status, cranial nerve deficits, and palsies) frequently persist. ${ }^{1}$ Longterm complications include the formation of a pseudoaneurysm or carotid cavernous fistula. Management of ICA injuries involves emergent hemostasis. Many techniques have been developed to manage ICA injury including controlled hypotension, ipsilateral and contralateral neck pressure, proximal control through neck dissection, surgical ligation, aneurysm clippings, direct suture repair, or muscle/fascia grafts, with varying degrees of success. Once hemorrhage has been controlled and resuscitation efforts are underway, patients often need emergent angiography and neuroradiological intervention, such as balloon occlusions or coil embolization. The size of received

February 18, 2017

accepted after revision

September 20, 2017
DOI https://doi.org/

10.1055/s-0037-1608635. ISSN 2193-6358.
๑) 2017 Georg Thieme Verlag KG
Stuttgart · New York

License terms

(1) $\circledast \circledast$ 
the arterial damage, its nature (small perforator avulsion vs. large, direct injury), and its site determine its treatment and the ability to control the injury while preserving the vessel. The ability to repair the ICA using endoscopic techniques is limited by the narrow surgical field and inability of suturing. Pressure application or bipolar cautery has been suggested in cases of small defects in the vessel wall. However, in cases of significant bleeding during EES, endovascular treatment is suggested as definitive treatment. The incidence of reported cases of ICA injury during EES is low, and thus development of an evidencebased management strategy is difficult. ${ }^{1}$ In this case report, we provide a detailed description of an endoscopic repair of ICA injury during EES.

\section{Case Report}

A 57-year-old otherwise healthy woman was referred to our clinic due to complaints of intense headaches, speech difficulties, and dysphagia for 2 weeks. Upon physical examination, the patient demonstrated slurred speech with tongue deviation toward the left. Neck examination and endoscopic flexible fiber optic evaluation were normal. No other neurological deficits were observed. Magnetic resonance imaging demonstrated an enhanced clival mass, $48 \times 36 \times 55 \mathrm{~mm}$, invading the right sphenoid sinus, occipital condyles, dens, and anterior arch of $\mathrm{C} 1$ ( - Fig. 1). The mass was pushing the pituitary gland upward, invading the parapharyngeal space as well as the upper, middle, and lower parts of the clivus with intradural extension through the midclival region pushing the pons and basilar trunk. Inferiorly, the mass invaded the occipital condyle on both sides, the anterior ring of $\mathrm{C} 1$ and the upper dens. Radiological features were consistent with chordoma. The patient was scheduled for an endonasal endoscopic resection.

\section{Surgical Technique}

The operation was performed under general anesthesia using an intraoperative frameless navigation. The surgery was conducted under electrophysiological monitoring. After inferior turbinate lateralization, middle turbinectomy, middle antrostomy, anterior and posterior ethmoidectomy, posterior septectomy, and elevation of a nasoseptal flap, the tumor was exposed in the sphenoid sinus. Using a high-speed coarse drill, the rostrum and clival tumor extensions were drilled out. The anterior wall of the sphenoid sinus was removed to provide better access to the tumor mass. Due to its rubbery consistency, the tumor could not be suctioned out. Instead, tumor removal was performed using Kerrison rongeur forceps and a drill. When the tumor was extirpated from the left sphenoid wall, bleeding from the left ICA in the cavernous segment was noticed. An immediate pressure with a neurosurgical gauze was applied over the defect. The operating team and the anesthesiologist were immediately notified. Packed cells and fresh-frozen plasma were delivered to the operating room and a second anesthesiologist joined the team. Vital signs were monitored carefully. Arterial blood was drawn to monitor blood gases and hematocrit levels. Neither hemodynamic compromise nor deviation from normal ranges of any of the monitored parameters was noted. Two attempts to correct the injury with Surgicel (Ethicon, West Somerville, New Jersey, United States) were unsuccessful. It was then decided to repair the injury with a temporalis muscle patch. At this point, the head and neck surgeon started prepping the temporalis area, while the neurosurgeon placed a neurosurgical gauze directly on the injured part of the left ICA applying local pressure for packing. Full control of the bleeding was maintained. Next, a $2 \times 2 \mathrm{~cm}$ muscle patch flap was quickly harvested from the left

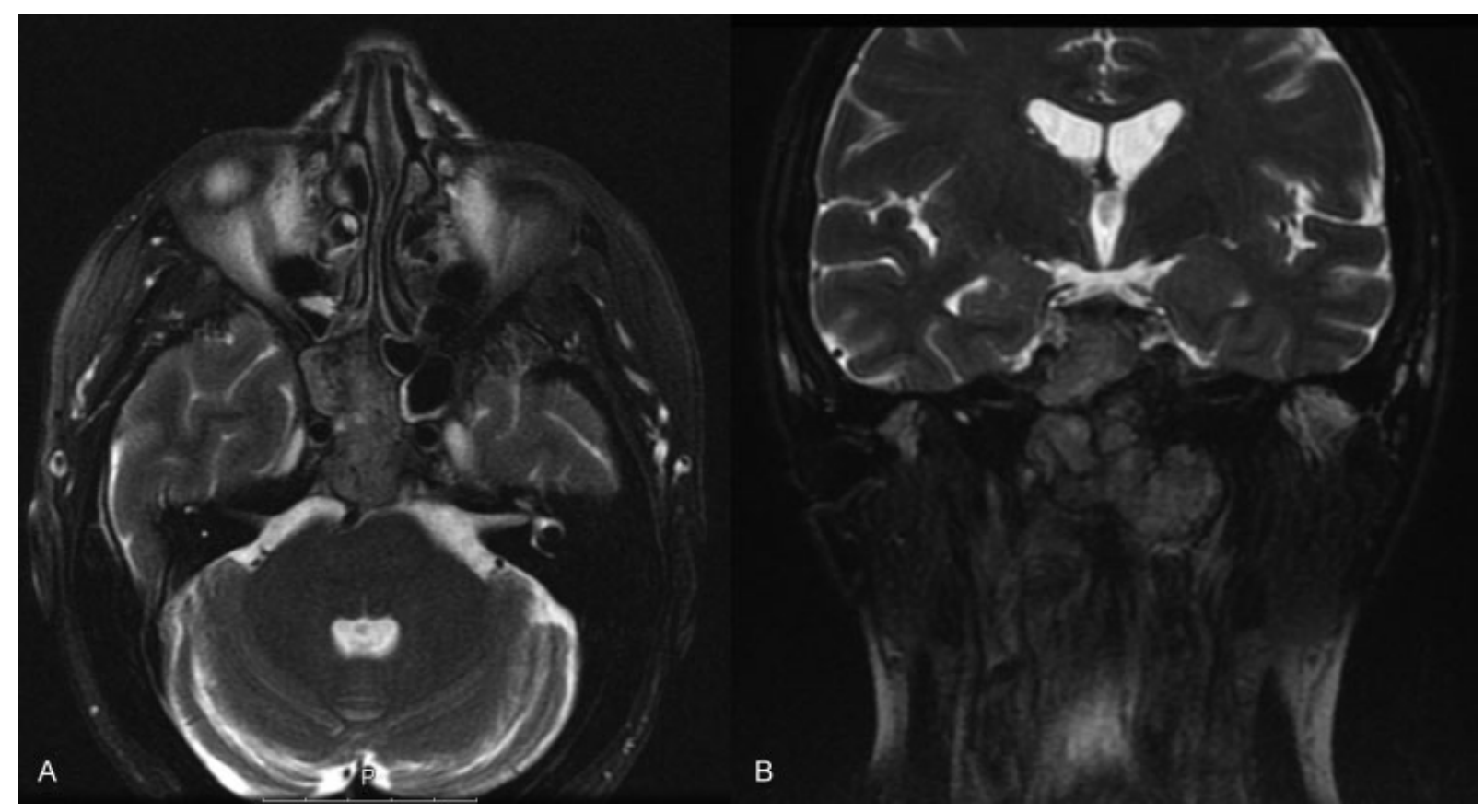

Fig. 1 Preoperative gadolinium-enhanced T2-weighted (A) axial and (B) coronal magnetic resonance imaging sections. An enhanced clival mass, $48 \times 36 \times 55 \mathrm{~mm}$, is shown, invading the right sphenoid sinus, the occipital condyles, the dens, and the anterior arch of C1. Radiological features were consistent with chordoma. 
temporalis muscle through a scalp incision at the superior temporal line in a coronal plane by the head and neck surgeon. After harvesting, the gauze was removed, and the muscle flap was inserted and positioned directly over the injured ICA. Gentle pressure was applied with overlying cotton patties for exactly 10 minutes. During that time, blood pressure was maintained above $100 / 60 \mathrm{~mm} \mathrm{Hg}$. After 10 minutes, the cotton patties were removed without evidence of residual bleeding. The total blood loss was $<250 \mathrm{~mL}$, and there was no hemodynamic compromise. Vital signs, including blood pressure, as well as laboratory results, including arterial blood gases, hemoglobin and hematocrit levels, were all within normal ranges. The patient received 1 unit of packed red blood cells (300 mL/unit) and 2 units of fresh-frozen plasma. After hemostasis was achieved, there was a question whether to discontinue the surgery. Based on the patient's stability during the time from the injury recognition until the bleeding was fully controlled, and given that a large portion of the tumor mass had already been removed prior to the injury, the operating team and anesthesiologist decided it was safe to proceed with the surgery. The operation continued for less than an hour. The degree of resection was also limited by the consistency of the tumor, which was firm and fibrotic. As most of the tumor was located below the vidian canal, the injury to the ICA did not limit the access to the rest of the tumor. Before injury, $50 \%$ of the tumor was resected, and by the end of the operation, $80 \%$ of the tumor had been removed. Throughout surgery, the right cranial nerve XII (CN XII) was monitored and preserved, while the left nerve was nonfunctional prior to surgery. Immediately after surgery, a computed tomography (CT) scan with contrast was performed, without evidence for pneumocephalus, pseudoaneurysm, or intracranial bleeding. After the operation, the patient was extubated uneventfully and transferred to the intensive care unit for observation. No postoperative complications were encountered. On 1-month follow-up, the patient was asymptomatic, maintained good outpatient oral intake, with great relief of preoperative complaints. There were no neurological deficits except for the known preoperative left CN XII palsy, which improved following surgery. The surgical wounds were intact, without evidence of infection or inflammation, and no local pain. Highquality CT angiogram was performed 6 weeks after surgery to rule out delayed pseudoaneurysm, an event with 50 to $60 \%$ prevalence. The CT angiogram showed no evidence for pseudoaneurysm nor narrowing of the artery. We believe that CT angiogram should be considered early after such an event. Pathological analysis of the specimen demonstrated clival chordoma. The patient was referred for adjuvant radiotherapy.

\section{Discussion}

Dissection around the cavernous, paraclival, and petrous segments of the ICA may be associated with vascular injury. ${ }^{1}$ The cavernous portion of the ICA has the highest rate of injury due to its intimate relationship with the sphenoid sinus. Thus, the identification of the ICA is essential when operating in the cavernous region. Gardner et $\mathrm{al}^{2}$ described their experience of seven ICA injuries in 2,015 endoscopic endonasal skull base cases over a 13-year period, reporting an incidence of $0.3 \%$. Most injuries involved the left ICA, and the most common diagnosis was chondroid neoplasm. Injury of the ICA might be associated with long-term neurological deficits. ${ }^{1}$ Therefore, when such a life-threatening complication occurs, prompt identification and intervention are crucial. Furthermore, proper preparation for such a complication is essential when planning operation on a tumor adjacent to the ICA. In light of the reported case, we propose and adopted the following changes to the operative protocol: first, in operations with high risk of ICA injury, the temporalis site should be prepped into the surgical field while setting up the surgery. Second, due to the high risk for vascular injury, we advise against the use of small cutting burrs for drilling near the ICA.

Several additional hemostatic techniques have been proposed and shown to be effective at achieving vascular control. These include muscle patch treatment (harvested sternocleidomastoid), as well as the use of Floseal (Baxter International; Deerfield, Illinois, United States), oxidized regenerated cellulose (Surgicel Nu-Knit; Ethicon), MicroFrance Wormald vascular clamps (Medtronic; Jacksonville, Florida, United States), and U-Clip anastomotic sutures (Medtronic) to suture the vascular defect. $^{3}$ Packing and placing adequate pressure have the potential to stop the bleeding; however, this may lead to complete occlusion of the artery. ${ }^{1}$ Thus, despite controlling the bleeding, the same complications of exsanguination may occur as a result of reduced blood flow to the brain. Applying pressure can lead to pseudoaneurysm formation, especially following endovascular recanalization. ${ }^{1}$ As Linskey et al reported in their literature review, abrupt ICA occlusion is associated with a stroke rate of up to $26 \%$ and a mortality rate of $12 \% .{ }^{4}$ Roski et al reported a $16.6 \%$ incidence of stroke in their long-term followup (average 12.5 years) after ligation of the ICA for an ICA aneurysm. ${ }^{5}$ Endovascular techniques (balloon occlusion and coil embolization by stent graft) are valid options if endonasal repair is not achieved. ${ }^{1}$ In such cases, following packing, the patient is delivered intubated to the endovascular suite.

\section{Conclusion}

There is no clear protocol to manage injury to the ICA during EES, although many have been proposed. The mortality and morbidity of an ICA injury during EES can be decreased if the team is properly trained for this event. The following steps are the basis of successful control of ICA bleeding: (1) early recognition of the ICA injury, (2) briefing of the team and preparations, (3) packing, (4) harvesting of temporalis muscle patch, (5) endoscopic placement of the muscle patch over the defect, and (6) gentle compression for 10 minutes. Using this technique, the continuity of the vessel is maintained and neurological complications can be avoided.

Note

This is an original article reviewed by all the authors and not under consideration for publication elsewhere.

Financial Disclosure

None. 
e128 Endoscopic Endonasal Repair of Internal Carotid Artery Injury Duek et al.

Conflict of Interest

None.

\section{References}

1 Chin OY, Ghosh R, Fang CH, Baredes S, Liu JK, Eloy JA. Internal carotid artery injury in endoscopic endonasal surgery: a systematic review. Laryngoscope 2016;126(03):582-590

2 Gardner PA, Tormenti MJ, Pant H, Fernandez-Miranda JC, Snyderman $\mathrm{CH}$, Horowitz MB. Carotid artery injury during endoscopic endonasal skull base surgery: incidence and outcomes. Neuro- surgery 2013;73(2, suppl operative):ons261-ons269, discussion ons269-ons 270

3 Valentine R, Wormald PJ. Controlling the surgical field during a large endoscopic vascular injury. Laryngoscope 2011;121(03):562-566

4 Linskey ME, Jungreis CA, Yonas $\mathrm{H}$, et al. Stroke risk after abrupt internal carotid artery sacrifice: accuracy of preoperative assessment with balloon test occlusion and stable xenon-enhanced CT. AJNR Am J Neuroradiol 1994;15(05):829-843

5 Roski RA, Spetzler RF, Nulsen FE. Late complications of carotid ligation in the treatment of intracranial aneurysms. J Neurosurg 1981;54(05):583-587 